\title{
Potential to reduce antibiotic use in secondary care: Single-centre process audit of prescription duration using NICE guidance for common infections 洸”
}

\author{
Authors: Neil Powell, ${ }^{\mathrm{A}}$ Jennie Stephens, ${ }^{\mathrm{B}}$ Rory Rule, ${ }^{\mathrm{C}}$ Ryan Phillips, ${ }^{\mathrm{D}}$ Megan Morphew, ${ }^{\mathrm{C}}$ Emma Garry, \\ Natasha Askaroff, ${ }^{C}$ Daniel Hiley, ${ }^{D}$ Charlie Strachan, ${ }^{E}$ Myles Sheehan ${ }^{C}$ and Caitlin McDonald ${ }^{C}$
}

\section{Introduction}

Antibiotic use drives antibiotic resistance. Reducing antibiotic use through reducing antibiotic course lengths could contribute to the UK national ambition to reduce total antibiotic use.

Methods

Medical notes were reviewed for patients who had received at least 5 days of antibiotic therapy; had been discharged in January 2019; and were from a 750-bed acute secondary care hospital in England. UK national guidelines were used to determine the excess antibiotic use in common medical infections: community-acquired pneumonia (CAP), hospitalacquired pneumonia (HAP), pyelonephritis, cellulitis, infective exacerbation of chronic obstructive pulmonary disease (IECOPD) and cholangitis.

Results

Four-hundred and twenty-three patients met the inclusion criteria. Of these, 307 (73\%) patient notes were retrieved and reviewed. One-hundred and seventy-three patients met the study case definitions, of which, 137 met short course criteria.

Potential antibiotic reductions (measured in defined daily doses) were identified for five of the six infections: $32 \%$ in CAP, $20 \%$ in HAP, $14 \%$ in IECOPD, $11 \%$ in cellulitis and $10 \%$ in pyelonephritis. These reductions were estimated to reduce total antibiotic use in medical specialties by $12.4 \%$, which equates to $3.6 \%$ of the hospital's total antibiotic use.

Conclusion

Clinical application of the evidence-based guidance for shorter antibiotic course lengths appears to be a valid strategy for reducing total antibiotic consumption.

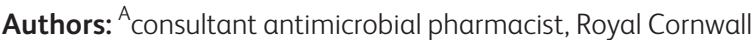
Hospital Trust, Treliske, UK and honorary clinical school fellow, University of Plymouth, Plymouth, UK; ${ }^{\mathrm{B}}$ consultant in intensive care and acute medicine, Royal Cornwall Hospital Trust, Treliske, UK;

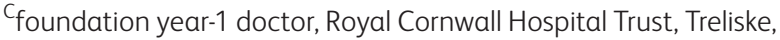

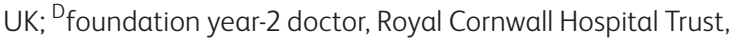
Treliske, UK; ${ }^{{ }}$core medical trainee-1 doctor, Royal Cornwall Hospital Trust, Treliske, UK
KEYWORDS: antimicrobial stewardship, optimise antibiotics, short course lengths, NICE clinical guidelines

DOI: 10.7861/clinmed.2020-0141

\section{Introduction}

Antimicrobial resistance is a global threat to healthcare and is on the UK National Risk Register. ${ }^{1,2}$ The use of antibiotics drives antibiotic resistance. ${ }^{3}$ Mitigation of the risks of antimicrobial resistance will require reductions in antibiotic use in humans and animals, in addition to improved understanding of the environmental spread, and containment of these organisms. ${ }^{4,5}$ The 5-year antimicrobial resistance (AMR) strategy includes a target of $15 \%$ reduction in antibiotic use by $2024 .{ }^{4}$

There is growing evidence demonstrating that shortened antibiotic course lengths, when compared with traditional longer courses, have comparable infection cure rates, relapse rates and mortality rates. ${ }^{6-9}$ Moreover, shorter antibiotic courses are associated with reduced adverse drug reactions, Clostridioides difficile and antibiotic-resistant infections. $6,7,10,11$

Due to the emergent data supporting short course antibiotic therapy, and the potential positive impact of this on antibiotic stewardship, the National Institute for Health and Care Excellence (NICE) have recently published a series of evidence-based antibiotic prescribing guidelines that recommend shorter course therapy for common infections treated in hospitals. ${ }^{12-16}$ This study sets out to determine the potential impact of implementing the guidance on shorter antibiotic course lengths on antibiotic exposure of medical patients at a district general hospital.

\section{Setting}

The hospital is a 750-bed acute secondary care hospital in the south-west of England with an electronic prescribing and medication administration (EPMA; JAC Computer Services (WellSky), Basildon, UK) system deployed in all inpatient areas, with non-mandated antibiotic indication field and non-mandated antibiotic stop date or review date field. Compliance with the trust requirement to document antibiotic indication and stop or review dates on the prescribing system is regularly audited 
and maintained at approximately $80 \%$ for both metrics. When measured in defined daily doses (DDD) of antibiotics adjusted for hospital admission rate, the study hospital (compared with other non-teaching hospitals in England) is ranked in the lowest quintile. $^{12,17}$

\section{Methods}

NHS ethics approval was not required as the study did not meet the Health Research Authority definition for research or the requirements for NHS Research Ethics Committee approval. The patient data were used in accordance with local NHS hospital policy.

The following NICE antibiotic prescribing guidelines for common infections managed by hospital medical specialties were identified and therefore included in this study: communityacquired pneumonia (CAP), hospital-acquired pneumonia (HAP), pyelonephritis, cellulitis, infective exacerbation of chronic obstructive pulmonary disease (IECOPD). ${ }^{13-16,18}$ In addition, the Tokyo guidelines on antibiotic prescribing in cholangitis, another frequently encountered medically managed infection, was also identified. ${ }^{19}$ Subsequent reference to 'NICE guidelines' assumes inclusion of the Tokyo cholangitis guideline.

In February 2019, pharmacy data analysts used the EPMA system to identify adult patients who were both admitted to, and discharged from, medical specialties at the study hospital between 01 and 31 January 2019, and who had received 5 or more days of antibiotic therapy. We chose 5 days because it was the shortest recommended course length in the NICE guidelines thereby identifying the cohort of patients where any extended antibiotic prescribing was occurring. For each patient we recorded age, gender, admission and discharge date, and discharging specialty. The medical notes were requested for all identified patients.

One of the study authors reviewed the medical notes of each patient and categorised the patient into one of the study infection groups if the pre-specified infection diagnostic criteria were met (HAP, CAP, IECOPD, cellulitis, pyelonephritis and cholangitis). Infections that were not included in this study (eg lower urinary tract infection (UTI)) and those patients with diagnoses that were included in the study but did not meet diagnostic criteria (eg CAP diagnosis in the medical notes but no evidence of focal consolidation or focal chest sounds on auscultation) were categorised as 'other'.

We defined our study population for each infection syndrome using infection-specific diagnostic criteria taken from the primary studies that informed the NICE antibiotic prescribing recommendations, shown in Table 1.

Patients diagnosed with one of the study infections who met the pre-defined infection syndrome diagnostic criteria were reviewed for appropriateness for short course antibiotic therapy as recommended by the infection specific NICE guideline. First, we determined whether the patient met any exclusion criteria for short course therapy using the criteria set out by NICE. If patients did not meet exclusion criteria for short course therapy, we reviewed their clinical course to determine whether the NICE criteria for short course therapy were met. Where a course length range was recommended in the NICE guidance, we selected the shortest course option as the audit standard (Table 1).

We collected antibiotic usage data and antibiotic duration of therapy for all patients who met the infection syndrome diagnostic criteria, regardless of whether they met the short course inclusion criteria or not. Antibiotic data included antibiotic name, dose and duration of antibiotic of each antibiotic and this was used to calculate the antibiotic DDD for each treatment episode. We calculated any excess DDD and any excess antibiotic days of therapy (DOT) for each patient that met the short course criteria.

We excluded patients discharged from the acute medical unit (AMU) because the HAP and CAP guidance advocate stopping at day 5 if they have shown adequate response to antibiotics. ${ }^{13,14}$ Patients discharged from AMU were unlikely to have inpatient stays of 5 days. However, patients discharged from the included medical specialties before day 5 , and who had met stability markers before discharge, we categorised as meeting 5-day course criteria: an assumption made that they would remain so after discharge.

Antibiotic usage data were extracted from the EPMA system for all medical patients and for all hospital patients, regardless of course length, both admitted and discharged in January 2019 from which we calculated antibiotic use in DDD. ${ }^{12}$ Excess antibiotic use due to prolonged antibiotic course lengths in medical patients were then quantified as a proportion of all antibiotic prescribing in medical specialties and also as a proportion of prescribing for all admitted patients over the study period. This enabled estimates to be made of the potential antibiotic usage reductions, in medical specialties and also the hospital, by optimising antibiotic course lengths in the six studied infections.

\section{Results}

Four-hundred and twenty-three patients met the inclusion criteria for notes retrieval, of which 307 (73\%) were received and reviewed against the case definitions and course length criteria defined in Table 1.

One-hundred and seventy-three patients met the infection case definitions. The median age was 77 (range 28-102). Gender data were missing for four patients but, of the remaining patients, $82 / 169(47 \%)$ were female.

Twenty-three patients out of $173(13.0 \%)$ met patient exclusion criteria (eg they were immunocompromised or had a critical care stay).

The 423 patients admitted and discharged from medical specialties in January 2019 received 6,123 DDD associated with their hospital stay (inpatient and discharge antibiotic consumption).

The 307 patients in whom we reviewed the medical notes received 3,904 DDD either during their stay or on discharge.

The excess DOT and resulting excess DDD attributed to each infection are shown in supplementary material S1. The proportion of antibiotic use that went beyond the optimal course length is shown as a percentage of the total antibiotic consumption in each infection group. The data are presented as excess antibiotic use in patients that met the inclusion criteria for short course therapy and also excess antibiotic use for both the included and excluded patients to give a potential excess antibiotic exposure figure for each infection. For example, in the CAP subgroup there is potential to reduce antibiotic usage by $44 \%$ in those patients that meet the criteria for short course therapy and by $32 \%$ in the entire CAP cohort if we include those patients that did not meet short course criteria. The median course lengths prescribed were within the range specified in the study hospital antibiotic prescribing guidelines at the time of the study except for IECOPD. 
Table 1. Patient infection syndrome-specific diagnostic criteria and infection-specific short antibiotic course inclusion and exclusion criteria

$\begin{array}{ll}\text { Infection } & \begin{array}{l}\text { Infection-specific diagnostic } \\ \text { criteria }\end{array} \\ \text { HAP \& CAP } & >18 \text { years old in hospital with } \\ & \text { CAP diagnosis (X-ray infiltrates or } \\ & \text { clinical evidence of pneumonia } \\ & \text { on examination and symptoms } \\ & \text { consistent with CAP (eg cough, } \\ & \text { fever, SOB or chest pain)). }\end{array}$

Cellulitis

Pyelonephritis

Kidney pain / tenderness in back or under ribs, nausea/ vomiting, shaking chills (rigors) or temperature $337.9^{\circ} \mathrm{C}$ or $\leq 36^{\circ} \mathrm{C}^{15}$

$>18$ years old with a diagnosis of IECOPD. ${ }^{18}$

$>18$ years old with cellulitis of trunk or extremity. Insect bites if a new acceleration of symptoms develop more than 48 hours after the initial insect bite. ${ }^{11}$

\section{Short course exclusion criteria}

HIV, chronic immunosuppression (solid organ transplant, splenectomy, neutropenia, prednisolone $>10 \mathrm{mg}$ od for $>30$ days and other immunosuppressive agents), pseudomonas or Staphylococcus aureus identified, requires chest drain, extrapulmonary infection, or critical care stay during admission. $7,13,14$

$n / a$

Bacteraemia, severe sepsis including septic shock, evidence of deep soft tissue infection (abscess, myositis, osteomyelitis, septic arthritis), infection requiring debridement at the site, animal or human bite wound cellulitis, neutropenia, diabetic foot infections with non-viable tissue suggesting vascular insufficiency, chronic cellulitis (symptoms present and stable $>2$ weeks at presentation), pregnancy or breast feeding, or severe renal insufficiency $(\mathrm{Cr}>354$ $\mathrm{mmol} / \mathrm{L}$ or eGFR $\left.<10 \mathrm{~mL} / \mathrm{min} / 1.73 \mathrm{~m}^{2}\right)^{11}$

Uncontrolled focus of infection. Positive blood culture with organism that warrants more than 7 days (ie $S$ aureus or Enterococcus).

\section{Cholangitis}

Suspected diagnosis is one item from $A$ plus one item from either $B$ or $\mathrm{C}$, and definite diagnosis is one item from $A$, one item from $B$ and one item from $\mathrm{C}^{20}$

A. Systemic inflammation:

A-1. fever and/or shaking chills

A-2. evidence of inflammatory response from laboratory data. B. Cholestasis:

B-1. jaundice

B-2. abnormal liver function tests from laboratory data.

C. Imaging:

C-1. biliary dilatation

$\mathrm{C}$-2. evidence of the aetiology on

imaging (stricture, stone, stent etc).

\section{Short course criteria}

Stop antibiotic treatment after 5 days unless microbiological results suggest a longer course is needed or the person is not clinically stable (fever in past 48 hours or more than one sign of clinical instability (systolic blood pressure $<90 \mathrm{mmHg}$, heart rate $>100$ beats/minute, respiratory rate $>24$ breaths/minute, arterial oxygen saturation $<90 \%$ or $\mathrm{PaO}_{2}<60 \mathrm{mmHg}$ on room air). ${ }^{13,14}$

All IECOPD for 5 days. ${ }^{18}$

Five to 7 days with longer courses (up to 14 days in total) based on clinical assessment ie appropriate response not evident. ${ }^{16}$

Response deemed appropriate if skin was less red, less hot, less tender or less oedematous by day $5 .^{11}$

Seven-day course of ciprofloxacin to treat acute pyelonephritis in men and nonpregnant women, or a longer 7-10-day course for beta-lactams due to a paucity of evidence for shorter course therapy with beta-lactams. NICE do not suggest stop criteria. As an indication of clinical stability at day 7 , patient needs to be haemodynamically stable and afebrile for 48 hours.

If bacteraemic with Enterococcus or Streptococcus then 14 days recommended (although risk of SBE is $0.3 \%$ ); liver abscess exclude. ${ }^{19}$
Once source of infection is controlled (ie effective biliary drainage) then duration is 4-7 days. ${ }^{19}$

$\mathrm{CAP}=$ community-acquired pneumonia; $\mathrm{Cr}$ = creatinine; eGFR = estimated glomerular filtration rate; $\mathrm{HAP}=$ hospital-acquired pneumonia; IECOPD = infective exacerbation of chronic obstructive pulmonary disease; NICE = National Institute for Health and Care Excellence; od $=$ once per day; $\mathrm{PaO}_{2}=$ partial pressure of oxygen; $\mathrm{SBE}=$ spontaneous bacterial endocarditis; $\mathrm{SOB}=$ shortness of breath. 
Table 2. Excess antibiotic use in defined daily doses and as a percentage of the total defined daily doses for the medical patients, including and excluding acute medical unit patients and all hospital patients who were both admitted and discharged in January 2019

\begin{tabular}{lllll} 
Patient group & $\begin{array}{l}\text { Number of } \\
\text { patients }\end{array}$ & DDD & Excess DDD & $\begin{array}{l}\text { Excess DDD as percentage } \\
\text { of total usage }\end{array}$ \\
Medical patients note review & 307 & 3,884 & 483 & $12.4 \%$ \\
Medical patients excluding AMU & 423 & 6,123 & Estimated $759.3(0.124 \times 6,123)$ & Estimated $12.4 \%$ \\
All hospital patients & 2,613 & 20,976 & Estimated 759.3 & Estimated $3.6 \%$ \\
\hline
\end{tabular}

$\mathrm{AMU}=$ acute medical unit; DDD = defined daily doses

Table 2 shows excess antibiotic use to be $12.4 \%$ across patients discharged from medical specialties.

One-hundred and thirty-four patients $(44 \%)$ were excluded due to an unclear diagnosis or because the patients' signs and symptoms of infection did not meet the pre-defined audit standard, or was not one of the study infections. Of these 134 patients, 31 (23\%) had a lower UTI; 29 (22\%) a lower respiratory tract infection (LRTI); 24 (18\%) were categorised as 'no evidence of bacterial infection identifiable in the medical notes'; 14 (10\%) had gastrointestinal infections; 12 (9\%) were categorised as 'unable to find patient notes corresponding to current admission'; nine $(7 \%)$ had a skin and skin structure infection; $8(6 \%)$ had an unclear diagnosis (source of infection unclear or multiple potential sources of infection); three ( $2 \%$ ) had a cardiovascular system infection; three ( $2 \%$ ) had an ear, nose or throat infection; and one $(1 \%)$ had a central nervous system infection.

\section{Discussion}

\section{Main findings}

We set out to determine the potential impact of implementing the evidence for shorter course lengths on total antibiotic use in medical patients in a UK district general hospital. Introducing shorter course lengths through implementation of the recently published national antibiotic guidance in common hospital infections has the potential to reduce antibiotic use in medical specialties by $12.4 \%$ which reduces the total hospital usage by $3.6 \%$.

Relative to NICE guidance, excess DOT in the included sample were identified in the case of CAP (31\%), HAP $(36 \%)$, IECOPD (23\%), cellulitis ( $49 \%)$ and pyelonephritis (19\%). There were 483 excess DDD across the studied infection groups. Of these, $383 / 483$ (79.3\%) were in the CAP and HAP groups combined. IECOPD accounted for $45 / 483(9.3 \%)$ of the excess DDD, cellulitis accounted for $26 / 483(5.4 \%)$ of the excess DDD, pyelonephritis accounted for $29 / 483(6.0 \%)$ of the excess DDD, and there was no evidence of excess antibiotic use in the treatment of cholangitis.

Shorter antibiotic courses reduce the potential for individual patient harm through reduced exposure to antibiotics, but it is often difficult to implement several concurrent antibiotic stewardship strategies due to resource constraints. These data show that antibiotic course lengths, with the exception of IECOPD, are in line with current local hospital guidelines. A focus on CAP and HAP, the most common infection diagnosis in this study and with over $70 \%$ of patients meeting short antibiotic course criteria, would deliver the majority of the potential reductions in antibiotic use in this study and contribute to the wider ambition to reduce antibiotic use by $15 \%$ by $2025 .{ }^{4}$ The publication of the NICE CAP and HAP antibiotic prescribing guidelines, which advocate short course therapy, should facilitate the implementation of short course antibiotic therapy for these infections, providing hospitals with the means to reduce total antibiotic consumption. ${ }^{13,14}$ This would reduce the risk of individual patient harm and wider societal harm through lower potential for antibiotic-associated side effects and the development of antibiotic resistance.

This study identifies potential opportunities to reduce antibiotic use further. Of the excluded 134 patients that did not meet diagnostic criteria for the study infections, 24/134 (18\%) were categorised as 'no evidence of bacterial infection identifiable in the medical notes' and 8/134 (6\%) as 'unclear diagnosis (source of infection is unclear or multiple potential sources of infection)'. For these patients without evidence of bacterial infection documented in the medical notes, or with an unclear diagnosis, the pre-72-hour antibiotic review may provide opportunities to review and stop unnecessary antibiotics and therefore warrants further study. ${ }^{20}$ Likewise, 29 patients with an LRTI diagnosis but did not meet CAP, HAP or IECOPD case definitions may meet diagnostic criteria for bronchitis or could be an incorrect LRTI diagnosis, and warrants further study.

\section{Strengths}

This study looked at a large data set and determined the potential to reduce antibiotic use at a patient level, an infection syndrome level and a hospital level. This has enabled an accurate estimate of the potential to reduce total antibiotic use in medical patients through implementation of short course antibiotic therapy guidance.

We were unable to obtain the medical notes for all identified patients which introduces the potential for selection bias. However, the 423 patients admitted and discharged from medical specialties in January 2019 received 6,123 DDD, averaging 14.5 DDD per patient. The 307 patients in whom we reviewed the medical notes received 3,904 DDD, averaging 12.7 DDD per patient. This shows that prescribing in the subpopulation for which notes were unavailable was not significantly different from those that we did review and, therefore, there was low potential for selection bias.

\section{Limitations}

This is a single centre study that looked only at 1 month of antibiotic prescribing in medical specialties. The findings, due to seasonality, may not be representative of annual antibiotic prescribing practices 
within the study hospital nor generalisable to other UK hospitals. The study hospital is a low antibiotic consuming hospital compared with other English hospitals. ${ }^{17}$ The median antibiotic course lengths for CAP and HAP in this study were 7 days; shorter than a 2017 study in the same hospital which showed median non-severe CAP and severe CAP course lengths to be 8.5 and 9 days, respectively. ${ }^{22}$ We believe the reductions in HAP and CAP course lengths seen in our hospital since 2017 to be due to local promotion of shorter antibiotic course lengths. The potential impact of short course antibiotic therapy on total antibiotic use may be greater in other hospitals than those seen in our study if work around antibiotic course length optimisation is yet to commence.

Although patients with an IECOPD diagnosis had a history of COPD and symptoms in keeping with a COPD exacerbation, we did not determine whether patients met criteria for antibiotic therapy. NICE suggests consideration for an antibiotic in acute exacerbation of COPD after taking into account the severity of symptoms, particularly sputum colour changes and increases in volume or thickness beyond the person's normal day-to-day variation. ${ }^{16}$ It is possible that not all patients in this study met criteria for requiring antibiotics and it warrants further study.

This study was carried out during the winter month of January and, therefore, respiratory tract infections are likely to be overrepresented in our study. Consequently, our estimates of the potential to reduce total annual antibiotic consumption may be an overestimate.

This study focused on excess total antibiotic use because of the link between total antibiotic use and antibiotic resistance. ${ }^{3}$ Other than the core measure of NICE-compliant reduction in DOT, we did not look at further quality indicators, such as whether unnecessarily broad spectrum antibiotic therapy was prescribed instead of narrower antibiotic regimens. While broader spectrum antibiotics are more likely to drive antibiotic resistance than narrower spectrum antibiotics' our local audit data demonstrate good compliance with local hospital antibiotic guidelines. ${ }^{3}$

This study set out to determine the opportunity to optimise antibiotic therapy through shortening antibiotic course lengths but did not quantify the opportunity to optimise antibiotic therapy through extending inappropriately short antibiotic course lengths. Shortening course lengths will reduce the likelihood of antibioticsrelated adverse drug events but too short will risk treatment failure.

\section{Future study}

This study did not include surgical patients treated for surgical infections or surgical patients treated for other non-surgical infections (eg HAP). Research in these patient groups is likely to identify further opportunities for reductions in antibiotic use through optimisation of antibiotic course lengths for surgical and non-surgical infections in surgical patients. ${ }^{23,24}$

Patients discharged from AMU and the emergency department were not included. There is likely an opportunity to reduce course lengths for non-severe infections discharged from these specialties.

Patients admitted under paediatrics, obstetrics or gynaecology were not included in this study. However, as NICE antibiotic guidelines for the common infections recommend short antibiotic courses for paediatric and young adult patients as well as adults, there are likely opportunities to optimise antibiotic use and reduce antibiotic consumption in these groups too.

\section{Conclusion}

Implementation of the NICE antimicrobial prescribing guidelines will reduce total antibiotic consumption in the studied UK hospital. The recently published NICE guidance on antibiotic therapy for common infections treated in secondary care can be used to facilitate this nationally. This may mitigate the harms associated with antibiotic therapy at a patient and population level.

\section{Supplementary material}

Additional supplementary material may be found in the online version of this article at www.rcpjournals.org/clinmedicine: S1 - Total antibiotic use in DDD and excess DDD.

\section{Acknowledgements}

We would like to thank Esther Dryden for extracting the antibiotic usage data from the EPMA system.

\section{References}

1 World Health Organization. Antimicrobial resistance: global report on surveillance 2014. WHO, 2014.

2 Cabinet Office. National Risk Register of Civil Emergencies: 2017 edition. Civil Contingencies Secretariat, 2017. https://assets. publishing.service.gov.uk/government/uploads/system/uploads/ attachment_data/file/644968/UK_National_Risk_Register_2017. pdf

3 Public Health England. English surveillance programme for antimicrobial utilisation and resistance (ESPAUR) report. London: PHE, 2019.

4 Global and Public Health Group, Emergency Preparedness and Health Protection Policy Directorate. Tackling antimicrobial resistance 2019-2024: The UK's five-year national action plan. Department of Health and Social Care, 2019. https://assets.publishing.service. gov.uk/government/uploads/system/uploads/attachment_data/ file/784894/UK_AMR_5_year_national_action_plan.pdf

5 Global and Public Health Group, Emergency Preparedness and Health Protection Policy Directorate. Contained and controlled: The UK's 20-year vision for antimicrobial resistance. Department of Health and Social Care, 2019. https://assets.publishing.service. gov.uk/government/uploads/system/uploads/attachment_data/ file/773065/uk-20-year-vision-for-antimicrobial-resistance.pdf

6 Spellberg B. The new antibiotic mantra-"shorter is better". JAMA Intern Med 2016;176:1254-5.

7 Uranga A, Espana PP, Bilbao A et al. Duration of antibiotic treatment in community-acquired pneumonia: A multicenter randomized clinical trial. JAMA Intern Med 2016;176:1257-65.

8 Yahav D, Franceschini E, Koppel $\mathrm{F}$ et al. Seven versus 14 days of antibiotic therapy for uncomplicated gram-negative bacteremia: a noninferiority randomized controlled trial. Clin Infect Dis 2019;69: 1091-8.

9 Hepburn MJ, Dooley DP, Skidmore PJ et al. Comparison of shortcourse ( 5 days) and standard ( 10 days) treatment for uncomplicated cellulitis. Arch Intern Med 2004;164:1669-74.

10 Tamma PD, Avdic E, Li DX, Dzintars K, Cosgrove SE. Association of adverse events with antibiotic use in hospitalized patients. JAMA Intern Med 2017:177:1308-15.

11 Stevens V, Dumyati G, Fine LS, Fisher SG, Van Wijngaarden E. Cumulative antibiotic exposures over time and the risk of clostridium difficile infection. Clin Infect Dis 2011;53:42-8.

12 WHO Collaborating Centre for Drug Statistics Methodology, Norwegian Institute of Public Health. Anti-infectives for systemic use. WHO, 2019. www.whocc.no/atc_ddd_index/?code=J04AB04 
13 National Institute for Health and Care Excellence. Pneumonia (community-acquired): antimicrobial prescribing: NICE guideline [NG138]. NICE, 2019.

14 National Institute for Health and Care Excellence. Pneumonia (hospital-acquired): antimicrobial prescribing: NICE guideline [NG139]. NICE, 2019.

15 National Institute for Health and Care Excellence. Pyelonephritis (acute): antimicrobial prescribing: NICE guideline [NG111]. NICE, 2018.

16 National Institute for Health and Care Excellence. Cellulitis and erysipelas: antimicrobial prescribing: NICE guideline [NG141]. NICE, 2019

17 Public Health England. AMR local indicators: Antibiotic prescribing: Total antibiotic prescribing DDD per 1000 admissions; by quarter and trust. PHE, 2019. https://fingertips.phe.org.uk/profile/amr-localindicators/data\#page/3/gid/1938132909/pat/158/par/NT_trust/ ati/118/are/REF/iid/93555/age/1/sex/4

18 National Institute for Health and Care Excellence. Chronic obstructive pulmonary disease (acute exacerbation): antimicrobial prescribing: NICE guideline [NG114]. NICE, 2018.

19 Gomi H, Solomkin JS, Schlossberg D et al. Tokyo Guidelines 2018 : antimicrobial therapy for acute cholangitis and cholecystitis. J Hepatobiliary Pancreat Sci 2018;25:3-16.
20 Kiriyama S, Takada T, Strasberg SM et al. New diagnostic criteria and severity assessment of acute cholangitis in revised Tokyo guidelines. J Hepatobiliary Pancreat Sci 2012;19:548-56.

21 Public Health England. Start smart - then focus: Antimicrobial stewardship toolkit for English hospitals. PHE, 2015.

22 Powell N, McGraw-Allen K, Menzies A et al. Identifying antibiotic stewardship interventions to meet the NHS England CQUIN: An evaluation of antibiotic prescribing against published evidencebased antibiotic audit tools. Clin Med 2018;18:276-81.

23 Sawyer RG, Claridge JA, Nathens AB et al. Trial of short-course antimicrobial therapy for intraabdominal infection. N Engl J Med 2015;372:1996-2005.

24 Leeds IL, Fabrizio A, Cosgrove SE, Wick EC. Treating wisely: the surgeon's role in antibiotic stewardship. Ann Surg 2017;265:871-3.

Address for correspondence: Mr Neil Powell, Pharmacy Department, Royal Cornwall Hospital, Treliske, Truro TR1 3LJ, UK.

Email: neil.powell2@nhs.net

Twitter: @neilthepcist 